\title{
Inhibition of eIF4F complex loading inhibits the survival of malignant glioma
}

\author{
QIU-FENG DONG ${ }^{*}$, ZHI-FENG YAN ${ }^{*}$, PENG-QI LI ${ }^{*}$, XIN YANG, JUN-LI HUO and HAI-NING ZHEN \\ Department of Neurosurgery, Xijing Hospital, Fourth Military Medical University, Xi'an, Shaanxi 710032, P.R. China
}

Received November 27, 2017; Accepted June 8, 2018

DOI: $10.3892 /$ or.2018.6587

\begin{abstract}
The eukaryotic initiation factor (eIF)4E-binding proteins (4E-BPs) regulate cap-dependent protein translation and control the assembly of the eIF4F complex. In the present study, a phosphorylation-deficient truncated 4E-BP2 (eIF4FD) was constructed into the eukaryotic expression vector pSecTag2, and the in vitro and in vivo effects on malignant glioma survival were determined through inhibiting eIF4F complex assembly. Cell cycle distribution analysis and TUNEL staining show that overexpression of eIF4FD suppressed cell proliferation and induced apoptosis in U251 cells. Western blotting showed that the cell cycle-related genes cyclin D1 and C-myc, and anti-apoptotic genes B-cell lymphoma 2 (Bcl-2), Bcl-extra large and survivin were reduced following the overexpression of eIF4FD. Furthermore, eIF4FD suppressed glioma vascularization via reductions in the expression of $\beta$-catenin and vascular endothelial growth factor. In the orthotopic xenograft model, the stable expression of eIF4FD in U251 cells attenuated cell growth and increased the rate of apoptosis. Accordingly, pSecTag2-PTD-eIF4FD injection via the tail vein of mice also lead to cell growth inhibition and the induction of apoptosis. Therefore, the study showed that phosphorylation-deficient truncated 4E-BP2 efficiently inhibited eIF4E and prevented the formation of the eIF4F complex, which further contributed to the inhibition of cell proliferation and vascularization, and the induction of apoptosis. Therefore, the 4E-BP2-based phosphorylation-deficient truncation designed in the present study may represent a novel approach for the targeted therapy of human malignant glioma though inhibition of the translation initiation complex.
\end{abstract}

Correspondence to: Dr Hai-Ning Zhen, Department of Neurosurgery, Xijing Hospital, Fourth Military Medical University, 127 Changle West Road, Xi'an, Shaanxi 710032, P.R. China

E-mail:zhenhn@fmmu.edu.cn

${ }^{*}$ Contributed equally

Key words: 4E-binding protein 2, eukaryotic initiation factor 4F complex, glioma

\section{Introduction}

Glioma is one the most common and aggressive types of brain cancer in humans, which is characterized by rapid proliferation, high invasion and genetic alterations, and is associated with a poor prognosis in patients $(1,2)$. Despite the significant advance in cancer treatments, the mortality rate remains high in malignant glioma (3). Therefore, it is important to investigate novel interventions or techniques for the treatment of malignant glioma.

Eukaryotic initiation factors (eIFs) are well known as key mediators of protein translation initiation in eukaryotic cells. Among them, eIF4E has been recognized as being key in the initiation and development of malignant tumors (4-6). eIF4E can be combined with the scaffolding protein eIF4G and the ATP-dependent RNA helicase eIF4A to form the eIF4F complex (7), which is the rate-limiting factor in a variety of cancer-related behaviors, including cell proliferation (4), apoptosis $(8,9)$ and angiogenesis $(10,11)$. Importantly, the deregulation of eIF4F activity is associated with the elevation of Myc in the prelymphomatous stage of E $\mu$-Myc lymphoma, whereas the inhibition of eIF4F causes the decrease in cycling pre-B/B cells and tumor onset delay (12). In addition, the inhibition of eIF4F complex formation either by inhibiting the eIF4E-eIF4G interaction or by targeting eIF4A, may lead to the death of cancer cells (13). These lines of evidence suggest that the eIF4F complex may be a promising molecular target for the treatment of malignant glioma.

The eIF4E binding proteins (4E-BPs) are a series of regulatory molecules involved in controlling eIF4F complex assembly. Following phosphorylation, 4E-BPs inhibit the activity of translation factor eIF4E through direct interactions (14-16). Among them, 4E-BP2 is one of the predominant 4E-BPs expressed in the brain (17), however, the associated functions remain to be fully elucidated. A previous study demonstrated that the interaction between eIF4E and 4E-BP2 inhibited eIF4F complex assembly and cap-dependent translation initiation (18). Therefore, 4E-BP2 may be a potential target for controlling cancer cell proliferation via affecting eIF4F complex assembly.

In the present study, based on the evidence that 4E-BPs bind to the dorsal side of eIF4E to prevent formation of the eIF4F complex, phosphorylation-deficient truncated 4E-BP2 (eIF4FD) was generated and constructed into the eukaryotic expression vector pSecTag2, and fused with the 
protein transduction domain (PTD), which has the potential to permeate cell membranes and deliver proteins into living cells $(19,20)$. This truncation was then transfected into the U251 cell line, and the alterations of biological characteristics and expression of cancer-related genes were determined in vitro and in vivo to fully address the effect and mechanism of inhibiting eIF4F complex assembly on malignant glioma.

\section{Materials and methods}

Cell culture. The human U251 glioma cells were purchased from the Chinese Academy of Sciences Cell Bank (Shanghai, China). The U87-MG cells were obtained from the American Type Culture Collection (ATCC; Manassas, VA, USA) and cultured at $37^{\circ} \mathrm{C}, 5 \% \mathrm{CO}_{2}$ in Dulbecco's modified Eagle's medium supplemented with $10 \%$ fetal bovine serum (FBS; HyClone Laboratories, Inc., South Logan, UT, USA), $100 \mathrm{U} / \mathrm{ml}$ of penicillin, $100 \mu \mathrm{g} / \mathrm{ml}$ of streptomycin and $2 \mathrm{mmol} / \mathrm{l} \mathrm{L-glutamine.}$

Plasmid constructs. Based on the GenBank sequence (NM_004096), upstream and downstream primers were synthesized using Primer 5.0 software (Premier Biosoft International, Palo Alto, CA, USA) (forward, 5'-AAAGGATCCACGTCC ACTAGCTGCCCCGATTCCC-3' and reverse, 5'-AAACTC GAGTTATTGTGCGTCATCGGGTATCTCT-3'. Restriction enzyme (BamHI and $\mathrm{XhoI}$ ) sites were added to the $5^{\prime}$ end of the upstream and downstream primers. Using the cDNA as a template, a phosphorylation-deficient truncated 4E-BP2 sequence with Flag-tag and $\mathrm{BamHI} / \mathrm{XhoI}$ restriction sites on each end was obtained by polymerase chain reaction (PCR). The thermocycling conditions were as follows: $95^{\circ} \mathrm{C}$ for $5 \mathrm{~min}$, then 35 cycles of $95^{\circ} \mathrm{C}$ for $15 \mathrm{sec}, 60^{\circ} \mathrm{C}$ for $15 \mathrm{sec}$, and $72^{\circ} \mathrm{C}$ for $30 \mathrm{sec}$ followed by $72^{\circ} \mathrm{C}$ for $10 \mathrm{~min}$. The PCR product was cloned into the pSecTag2-PTD vector (Thermo Fisher Scientific, Inc., Waltham, MA, USA). As a result, the recombinant expression vector pSecTag2-PTD-eIF4FD was obtained.

Transfection. The day preceding transfection, the U251 or U87-MG cells in the logarithmic growth phase were trypsinized, counted and seeded in 6-well plates at the density of $1 \times 10^{6}$ cells/well. When the cells were $80 \%$ confluent, the medium was replaced with serum-free RPMI-1640 medium (Hyclone Laboratories), and pSecTag2 and pSecTag2-PTD-eIF4FD were transfected into the culture overnight according to the manufacturer's protocol (Lipofectamine ${ }^{\mathrm{TM}} 2000$ for plasmid delivery). To set up the U251 cell line stably expressing eIF4FD, the $5 \times 10^{5}$ transfected cells were seeded in 12-well plates and cultured in a complete medium containing G418 ( $200 \mu \mathrm{g} / \mathrm{ml})$. The positive clones were selected and confirmed by western blot analysis.

Cell cycle distribution analysis. The cells were collected (detached by $0.25 \%$ trypsinization without EDTA) at $0,12,24$ and $48 \mathrm{~h}$ post-transfection, and then washed twice with phosphate-buffered saline (PBS) and centrifuged at 12,000 x g for 5 min at $4^{\circ} \mathrm{C}$. The pellet was fixed with $70 \%$ ethanol for $1 \mathrm{~h}$ at $4^{\circ} \mathrm{C}$. Following washing with PBS, the cells were resuspended with propidium iodide (PI) solution $(0.05 \mathrm{mg} / \mathrm{ml})$ containing RNase and incubated at room temperature in the dark for
30 min. DNA content was then analyzed using the FC 500 flow cytometer (Beckman Coulter, Inc., Brea, CA, USA).

TUNEL staining. Briefly, the U251 cells were fixed with $4 \%$ formaldehyde solution for $20 \mathrm{~min}$ at room temperature. The cells were then permeabilized with $0.2 \%$ Trition $\mathrm{X}-100$ for $5 \mathrm{~min}$. The cells were labeled with fluorescein TUNEL reagent mixture for $60 \mathrm{~min}$ at $37^{\circ} \mathrm{C}$ according to the manufacturer's protocol (Promega Corp., Madison, WI, USA), and the nuclei were counterstained with 4',6-diamidino-2-phenylindole. Subsequently, the numbers of TUNEL-positive cells were counted under fluorescence microscopy.

Co-immunoprecipitation. Whole U251 cell lysates were obtained by resuspending U251 cell pellets in RIPA buffer containing $150 \mathrm{mM} \mathrm{NaCl}, 20 \mathrm{mM}$ Tris- $\mathrm{HCl}$ (pH 7.4), $5 \mathrm{mM}$ EDTA, 1\% NP-40, $1 \%$ nadeoxycholate, $0.1 \%$ SDS, $1 \mathrm{mM}$ PMSF, $20 \mathrm{mg} / \mathrm{ml}$ leupeptin, $20 \mathrm{mg} / \mathrm{ml}$ aprotinin and $3 \mathrm{mg} / \mathrm{ml}$ pepstatin A. The lysates were incubated overnight with Flag antibody (cat. no. ab49763; Abcam, Cambridge, UK) at $4^{\circ} \mathrm{C}$ prior to being absorbed with protein A/G PLUS agarose beads. The precipitated immunocomplexes were released by boiling with 2X SDS electrophoresis sample buffer and were prepared for western blot analysis.

Western blot analysis. For western blot analysis, the cells were washed twice with ice-cold PBS and lysed with RIPA buffer (Beyotime Institute of Biotechnology, Haimen, China) on ice. Following centrifugation at $12,000 \mathrm{xg}$ for $10 \mathrm{~min}$ at $4^{\circ} \mathrm{C}$, the protein concentration in the supernatant was analyzed using a bicinchoninic acid assay kit (Thermo Fisher Scientific, Inc.). The total protein $(20 \mu \mathrm{g})$ from each sample was separated by SDS-PAGE and transferred onto a polyvinylidene difluoride membrane (EMD Millipore, Bedford, MA, USA). The membrane was then blocked with a TBS solution containing $5 \%$ non-fat dry milk at room temperature for $1 \mathrm{~h}$, followed by incubation with the following corresponding primary antibodies: B-cell lymphoma 2 (Bcl-2; dilution 1:1,000; cat. no. ab32124; Abcam, Cambridge, MA, USA), Bcl-extra large (Bcl-xL: 1:1,000; cat. no. ab32370; Abcam), C-myc (1:1,000; cat. no. ab32072; Abcam), cyclin D1 (dilution 1:1,000; cat. no. 2922; Cell Signaling Technology, Inc., Beverly, MA, USA), survivin (dilution 1:1,000; cat. no. ab76424; Abcam), $\beta$-actin (dilution 1:500; cat. no. sc-517582; Santa Cruz Biotechnology, Inc., Santa Cruz, CA, USA), $\beta$-catenin (dilution 1:500; cat. no. sc-65480; Santa Cruz Biotechnology, Inc.) overnight at $4^{\circ} \mathrm{C}$. The following day, the membrane was washed with Tris-buffered saline with Tween-20 (0.05\%; TBST) three times ( 5 min each), and then incubated with HRP-labeled secondary antibody at $37^{\circ} \mathrm{C}$ for $1 \mathrm{~h}$ (dilution 1:5,000; cat. nos. BA1050 or BA1054; Boster Bio, Pleasanton, CA, USA). Subsequently, the membrane was washed with TBST three times and then developed using an enhanced chemiluminescence reagent. $\beta$-actin was used as a normalization control.

Glioma xenograft model. Male 8-9-week-old BALB/c (nu/nu) nude mice (20-25 g) were purchased from the Animal Center of the Fourth Military Medical University (Xi'an, China), and housed under controlled laboratory conditions on a 12-h light/12-h dark cycle at room temperature with access to water 
and food ad libitum. All procedures were in accordance with the NIH Guide and were approved by the Ethics Committee of the Fourth Military Medical University. The surgical procedure was performed as previously described $(21,22)$. Briefly, the mice were mounted on a stereotaxic instrument following anesthesia with sodium pentobarbital (intraperitoneal injection, $50 \mathrm{mg} / \mathrm{kg}$ ). Following skin incision, a hole with a diameter of $3 \mathrm{~mm}$ was drilled on the skull at the site of $1.0 \mathrm{~mm}$ anterior to the anterior fontanel and $2.0 \mathrm{~mm}$ lateral to the sagittal suture. A microsyringe needle (Hamilton Bonaduz AG, Bonaduz, Switzerland) was inserted through the hole to a depth of $3 \mathrm{~mm}$ beneath the dura. Subsequently, 5x10 $\mathrm{U} 251$ cells in $2 \mu \mathrm{l}$ PBS were injected slowly. Images of the mice were captured and the tumor sizes were measured using the high-efficiency CCD camera system (Xenogen Corp., Alameda, CA, USA) at $\sim 5$ min post-injection with $100 \mu \mathrm{g}$ of luciferin substrate (PerkinElmer, Inc., Waltham, MA, USA) in PBS without calcium or magnesium (Corning Inc., Manassas, VA, USA) as previously described (23).

Plasmid injections via the tail vein. Hydrodynamic tail vein injections were performed as described. The first plasmid delivery was performed on the second day following U251 cell injection in nude mice. The pSecTag 2 or pSecTag2-PTD-eIF4FD plasmid $(50 \mu \mathrm{g})$ was diluted in $1.8 \mathrm{ml}$ of QR buffer (Mirus Bio, Madison, WI, USA) and injected into the tail vein in $5 \mathrm{sec}$ via a 27 -gauge, 0.45 -inch needle. The animals were placed into a restraining device and injected without anesthesia. The plasmids were administered twice a week for 4 weeks. The tumor size was measured with CCD camera system every 5 days. The animal experiments were terminated at 30 days, and all nude mice were sacrificed. The tumor grafts were isolated for immunohistochemistry (IHC), western blot analysis and TUNEL staining assays.

IHC analysis. The IHC was performed using the avidin-biotin-peroxidase method. Tissues were fixed in $4 \%$ paraformaldehyde for $12 \mathrm{~h}$ at room temperature, embedded in paraffin and cut into $5-\mu \mathrm{m}$ sections. All sections were deparaffinized in xylene and dehydrated using a concentration gradient of alcohol, following which endogenous peroxidase activity was blocked using $0.5 \% \mathrm{H}_{2} \mathrm{O}_{2}$ in methanol for $10 \mathrm{~min}$. When non-specific binding was blocked, the slides were incubated with anti-PCNA antibody (dilution 1:200; cat. no. sc-25280; Boster Bio) overnight at $4^{\circ} \mathrm{C}$. Following rinsing with TBS for $15 \mathrm{~min}$, biotinylated goat anti-rabbit IgG (dilution 1:400; Sigma; EMD Millipore) was incubated with the sections for $1 \mathrm{~h}$ at room temperature and detected with a streptavidin-peroxidase complex. The brown color indicative of peroxidase activity was developed by incubating with $0.1 \%$ 3,3-diaminobenzidine (Sigma; EMD Millipore) in PBS with $0.05 \% \mathrm{H}_{2} \mathrm{O}_{2}$ for $5 \mathrm{~min}$ at room temperature. The appropriate positive and negative controls were included in each IHC run under a phase contrast microscopy (Olympus IX50; Olympus Corp., Tokyo, Japan).

Statistical analysis. All data are presented as the mean \pm standard deviation. SPSS 16.0 statistical software (SPSS, Inc., Chicago, IL, USA) was used to perform the statistical analysis. Comparisons of the mean values were performed by one-way analysis of variance (ANOVA), followed by Duncan's multiple-range test. $\mathrm{P}<0.05$ was considered to indicate a statistically significant difference.

\section{Results}

eIF4FD inhibits cell cycle and suppresses proliferation in glioma cells. The present study first confirmed the protein overexpression of eIF4FD in pSecTag2-PTD-eIF4FD-transfected U251 cells (Fig. 1A). To determine the effect of eIF4FD on cell survival, flow cytometry was performed in U251 cells following pSecTag2-PTD-eIF4FD transfection. The expression of eIF4FD led to a marked decrease in the DNA synthesis phase (S) and an increase in the $G_{0}-G_{1}$ phase in the U251 cells (Fig. 1B). Accordingly, cell cycle-related proteins cyclin D1 and C-myc were reduced $12 \mathrm{~h}$ following pSecTag2-PTD-eIF4FD transfection, and were significantly decreased at 24 and $48 \mathrm{~h}$ (Fig. 1C-E). The reductions of cyclin D1 and C-myc were also observed in U87-MG cells following expression of eIF4FD (Fig. 1F). Therefore, the overexpression of eIF4FD inhibited cell cycle progression and proliferation in the glioma cells. To determine whether the effect of eIF4FD on cell survival was through inhibition of the eIF4F complex, the interaction of eIF4FD with eIF4E following pSecTag2-PTD-eIF4FD transfection was examined. The co-immunoprecipitation assay showed that eIF4FD directly interacted with eIF4E but did not affect the expression of eIF4E (Fig. 1G and H). Therefore, eIF4FD inhibited the eIF4F complex via direct interaction with eIF4E.

eIF4FD induces glioma cell apoptosis and vascularization. To determine whether pSecTag2-PTD-eIF4FD has a tumor suppressive effect through inducing cell death, the expression levels of anti-apoptotic genes Bcl-2, Bcl-xL and survivin were examined. Their expression decreased in the pSecTag2-PTD-eIF4FD-transfected cells, particularly at $48 \mathrm{~h}$ (Fig. 1C and I-K), indicating that inhibiting eIF4F complex formation was critical for the induction of apoptosis through regulating the expression of $\mathrm{Bcl}-2, \mathrm{Bcl}-\mathrm{xL}$ and survivin. Furthermore, the vascularization associated genes $\beta$-catenin and VEGF were also decreased following the overexpression of eIF4FD (Fig. 1C, L and M). Accordingly, the decreases in of Bcl-2, Bcl-xL, survivin, $\beta$-catenin and VEGF were also observed in U87-MG cells following pSecTag2-PTD-eIF4FD transfection (Fig. 1F). The cell apoptotic rate was also detected through TUNEL staining of U251 cells (Fig. 2). As expected, cells transfected with pSecTag2-PTD-eIF4FD resulted in a significant increase in the number of TUNEL-positive cells. Therefore, the eIF4FD regulation of cell apoptosis and vascularization were shown to exist generally in glioma cells.

eIF4FD suppresses tumor growth of glioma cells in nude mice. To further investigate the effect of inhibiting eIF4F complex assembly via the expression of eIF4FD on cell growth, U251 cells with stable expression of eIF4FD were established through transfection with pSecTag2 or pSecTag2-PTD-eIF4FD, and their effects on tumorigenicity were determined via the subcutaneous glioma model in nude mice. The mice injected with U251 cells stably expressing eIF4FD developed tumors more slowly than the other groups (Fig. 3A) $(\mathrm{P}<0.01$, vs. control; 
A

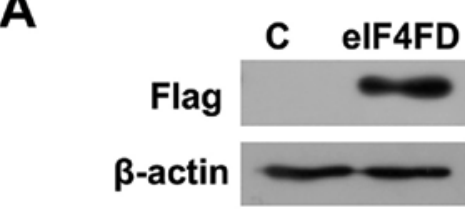

B

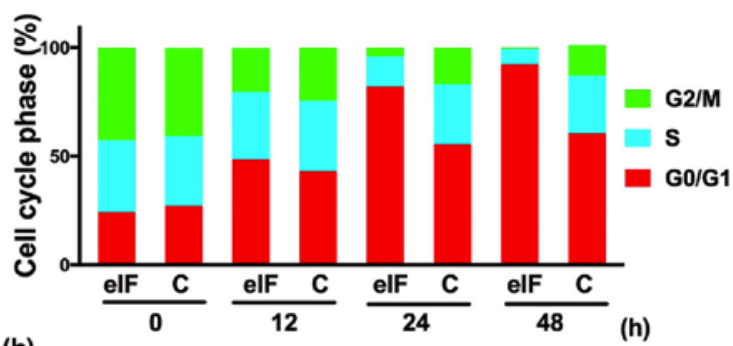

C $\frac{0}{\text { elF } \quad C} \frac{12}{\text { elF }}$ C $\frac{24}{\text { elF }}$ C $\frac{48 \quad(h)}{\text { elF }}$

C-myc

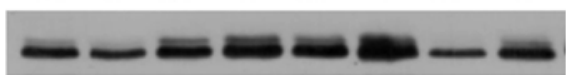

Cyclin D1

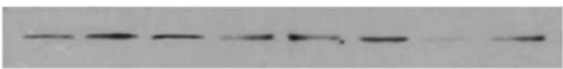

$\mathrm{Bcl}-2$

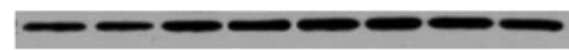

$\mathrm{Bcl}-\mathrm{xL}$

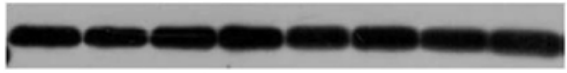

Survivin

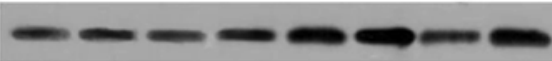

$\beta$-catenin

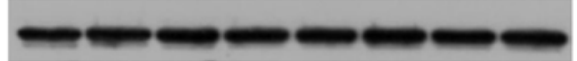

VEGF

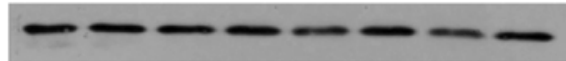

$\beta$-actin

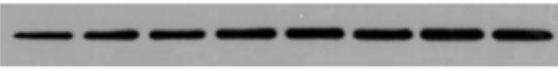

$\mathbf{F}$

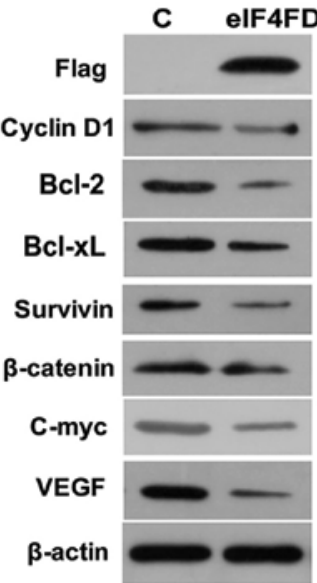

G

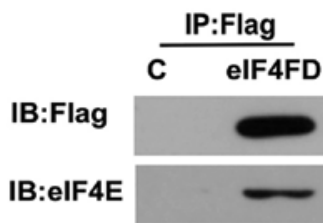

D

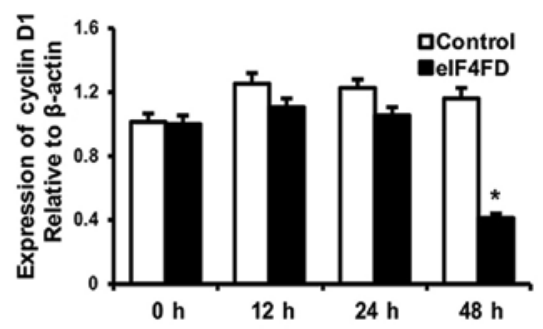

E

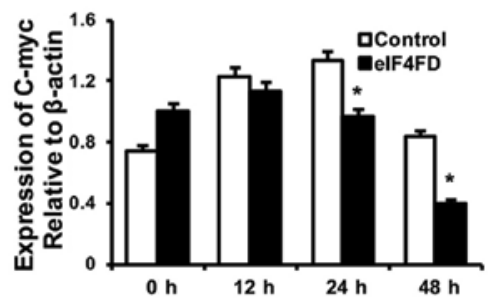

H
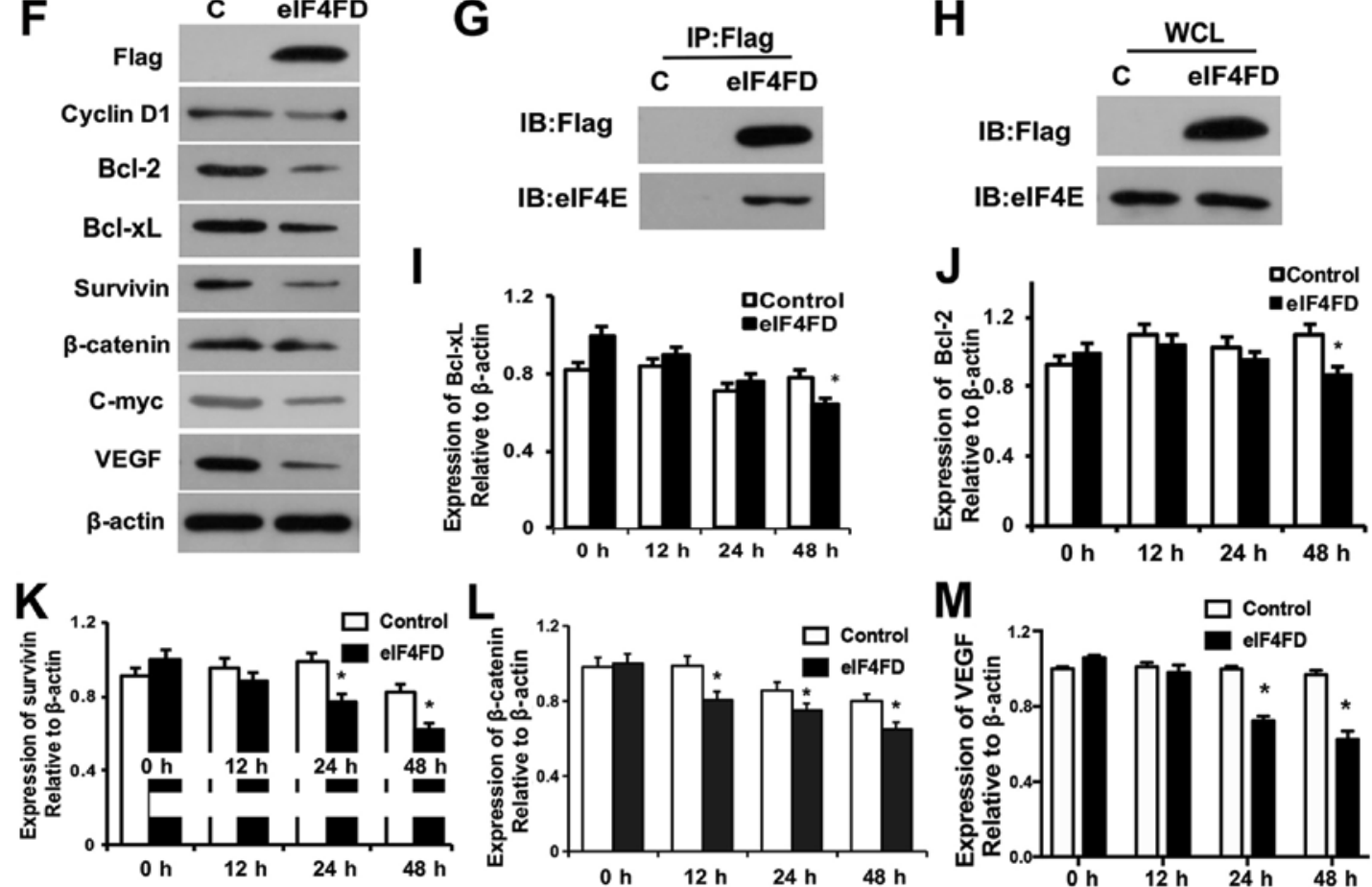

Figure 1.eIF4FD inhibits cell cycle and suppresses proliferation in glioma cells. (A) U251 cells were transfected with pSecTag2 control or pSecTag2-PTD-eIF4FD The indicated proteins were determined by western blot analysis $48 \mathrm{~h}$ post-transfection. (B) At $0,12,24$ and $48 \mathrm{~h}$ post-transfection, cells were collected and subjected to flow cytometric analysis. (C) Cell lysates were analyzed for the indicated protein levels by western blot analysis. $\beta$-actin was used as a loading control. Expression levels of (D) cyclin D1 and (E) C-myc were quantified using ImageJ software and normalized to $\beta$-actin. " $\mathrm{P}<0.05$ (F) U87 cells were transfected with pSecTag2 or pSecTag2-PTD-eIF4FD. The indicated proteins were determined by western blot analysis $48 \mathrm{~h}$ post-transfection. U251 cells with or without pSecTag2-PTD-eIF4FD transfection were collected for the co-immunoprecipitation assay. (G) Anti-Flag immunoprecipitates and (H) WCL were resolved by SDS-PAGE and analyzed by western blot analysis with anti-Flag antibody or anti-eIF4E antibody. Cell lysates were subjected to western blot analysis to determine levels of (I) Bcl-xL, (J) BCL2, (K) survivin, (L) $\beta$-catenin and (M) VEGF. WCL whole-cell lysate. C, control; eIF, eukaryotic initiation factor; PTD, protein transduction domain; BCL, B-cell lymphoma 2; Bcl-xL, BCL-extra large; VEGF, vascular endothelial growth factor.

$\mathrm{P}<0.05$, vs. vector). The results of the $\mathrm{IHC}$ assay showed that the positive rates of PCNA and Bcl-2 expression were decreased (Fig. 3B), indicating that the expression of eIF4FD significantly inhibited tumor growth and induced apoptosis in 


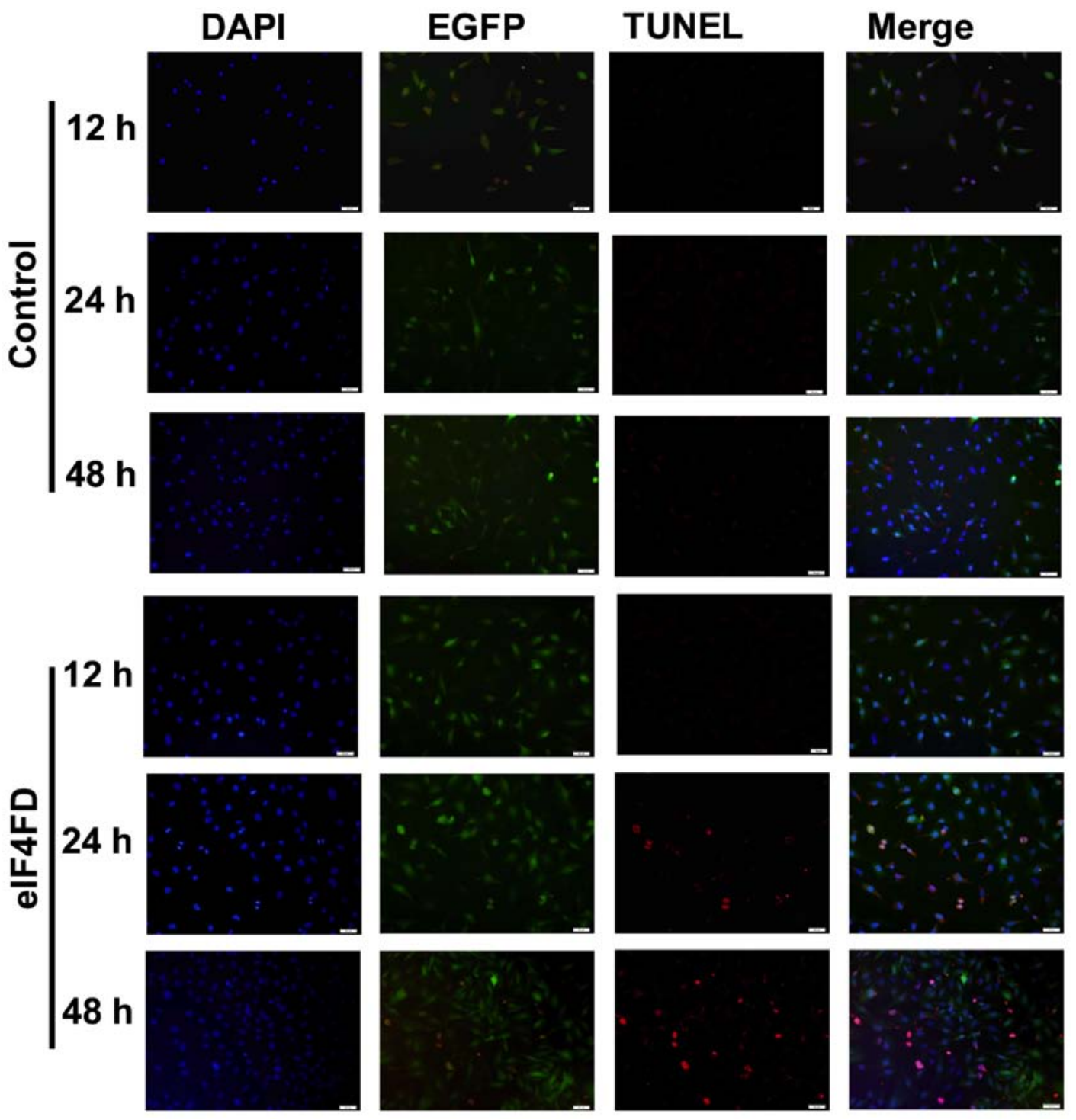

Figure 2. eIF4FD induces glioma cell apoptosis and vascularization. U251 cells were transfected with pSecTag2-PTD-eIF4FD and its empty vector, respectively. At $0,12,24$ and $48 \mathrm{~h}$ post-transfection, the cells were collected to perform TUNEL staining. Scale bar, $10 \mu \mathrm{m}$. eIF, eukaryotic initiation factor; PTD, protein transduction domain; DAPI, 4',6-diamidino-2-phenylindole.

solid tumors. Accordingly, TUNEL staining showed that the expression of eIF4FD promoted the apoptotic cell rates in the tumor tissues (Fig. 3C).

pSecTag2-PTD-eIF4FD injection via the tail vein represses glioma growth in vivo. To evaluate the therapeutic potential of eIF4FD via inhibiting eIF4F complex assembly, pSecTag2-PTD-eIF4FD was injected via the tail vein of nude mice with glioma xenografts. Treatment with pSecTag2-PTD-eIF4FD had a significant inhibitory effect on tumor growth compared with the control or empty vector groups $(\mathrm{P}<0.05$; Fig. 4A). Accordingly, PCNA and Bcl-2 were reduced and TUNEL staining positive cell enrichment were observed in glioma tissues (Fig. 4B and C). Genes associated with cell cycle, apoptosis and vascularization were also decreased in glioma grafts following pSecTag2-PTD-eIF4FD injection (Fig. 4D). Therefore, inhibiting eIF4F complex assembly by eIF4FD offers therapeutic potential in the treatment of gliomas.

\section{Discussion}

The eIF4F complex has attracted increasing attention as a molecular target for malignant tumors. The eIF4F complex is composed of eIF4E, eIF4G and eIF4A. Progress has been made in elucidating the association of eIF4E with the occurrence and development of malignant tumors in previous years. Studies have shown that eIF4E was expressed at high levels in nasopharyngeal carcinoma (NPC) and the overexpression of eIF4E promoted NPC growth and cell cycle progression (24). eIF4E silencing or inhibition reduced the invasiveness and metastatic capability of breast cancer cells (25). The inhibition of eIF4E by small interfering RNA induced cell cycle arrest and suppressed the cell growth and migration of MDA-MB-231 TN breast cancer cells (26). Furthermore, the overexpression of eIF4E in renal clear cell carcinoma was correlated with the presence of phosphorylated (p)4E-BP1. The combined expression of p4E-BP1 and eIF4E was associated with significantly poorer disease-free survival rate (27). Therefore, eIF4E and 

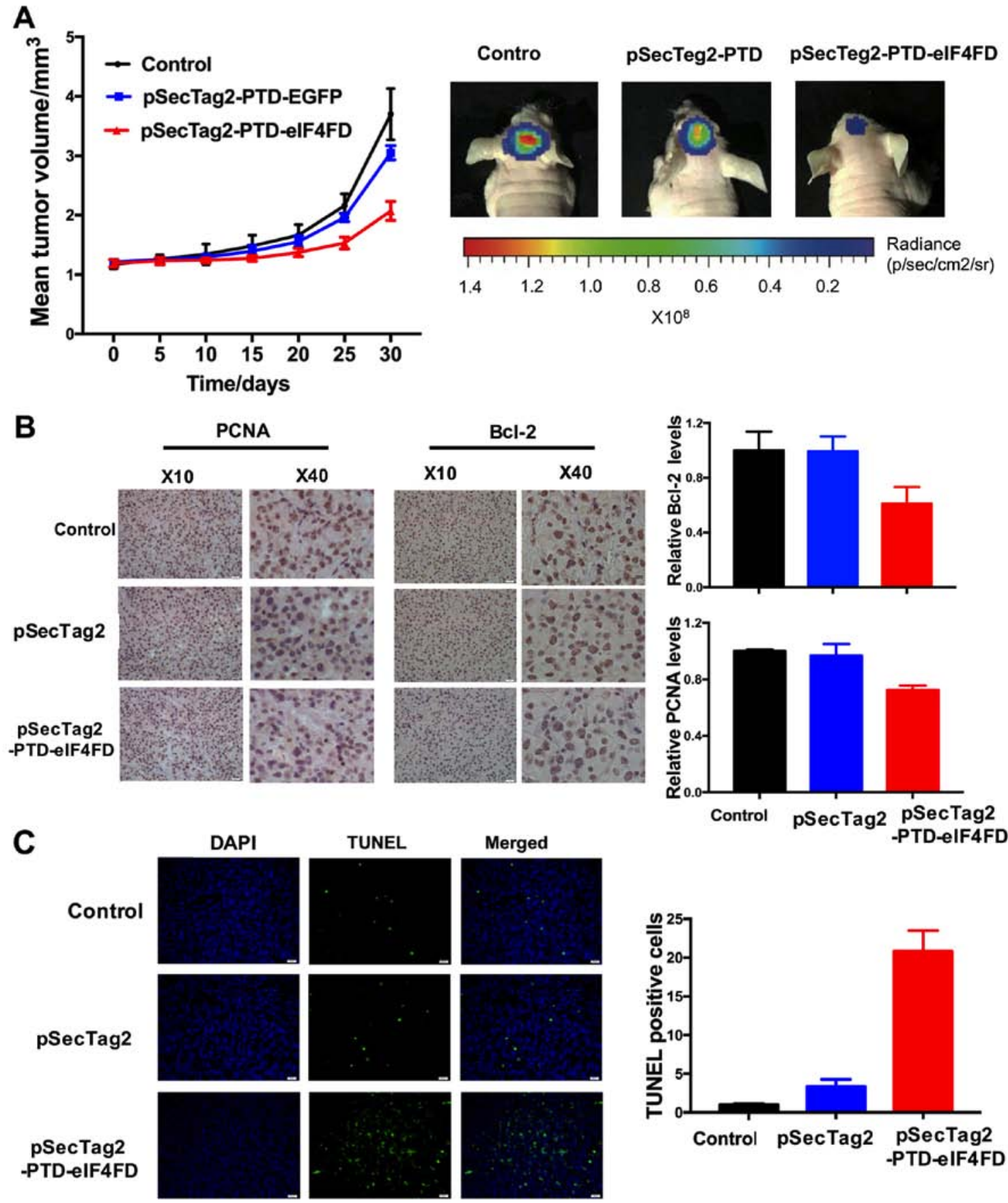

Figure 3. eIF4FD suppresses tumor growth of glioma cells in nude mice. U251 cells with or without eIF4FD stable expression were injected into nude mice. Tumor growth was monitored over 30 days. Tumor volume was assessed every 5 days. (A and B) Growth curve was drawn according to tumor volume. Expression of PCNA and BCL-2 were detected using an immunohistochemical assay. (C) TUNEL staining is used to detect the apoptosis of tumor tissues. Scale bar, $10 \mu \mathrm{m}$. The experimental results were analyzed by image analysis and statistical analysis. PCNA, proliferating cell nuclear antigen; eIF, eukaryotic initiation factor; PTD, protein transduction domain; BCL2, B-cell lymphoma 2; DAPI, 4',6-diamidino-2-phenylindole.

4E-BPs may be considered as a potential target for cancer therapy.

In our previous studies, U251 cells were treated with the eIF4E inhibitor 4EGI-1, and its suppressive effect on cell growth was observed (28). It has been confirmed that the initiation of the translation of cyclin D1 and C-myc are dependent on the eIF4F complex, which directly promotes the proliferation of cancer cells (4). 4E-BPs act as a post-transcriptional regulator to inhibit mRNA translation through interacting with eIF4E (29). The present study showed that the expression of eIF4FD, phosphorylation-deficient truncated 4E-BP2, is associated with cell cycle arrest in $G_{0} / G_{1}$ phase, which may be due to the downregulation of growth factors associated with the signal transduction pathway. The expression of cyclin D1 and C-myc were determined, which were shown to decrease in glioma cells following pSecTag2-PTD-eIF4FD transfection. Therefore, cell growth inhibition by pSecTag2-PTD-eIF4FD-mediated inhibition of the eIF4F complex may be the result of a reduction in cyclin D1 and C-myc, although the exact mechanism remains to be elucidated. There is evidence showing that activation 
A
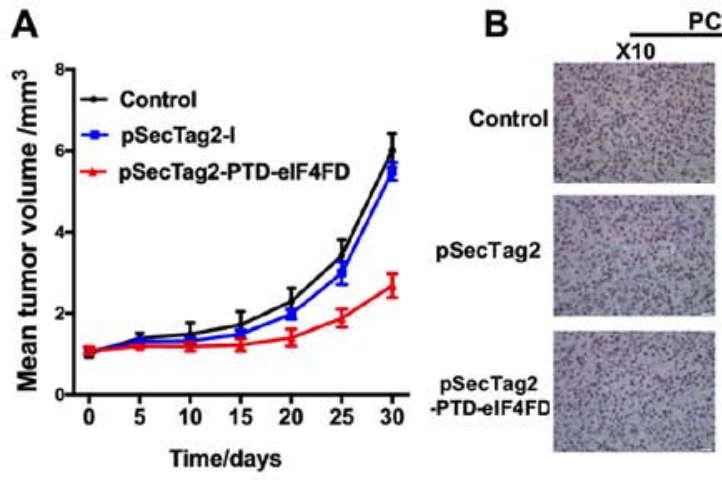

C

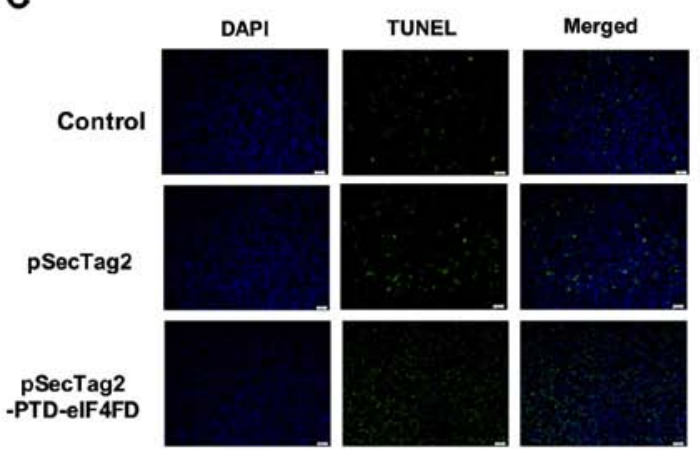

PCNA

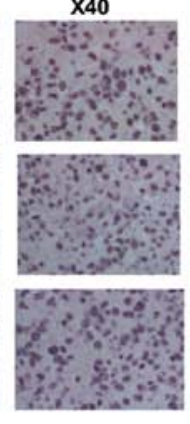

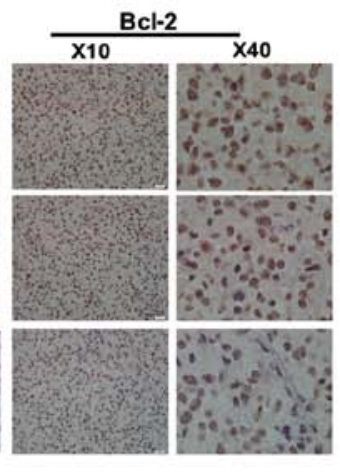
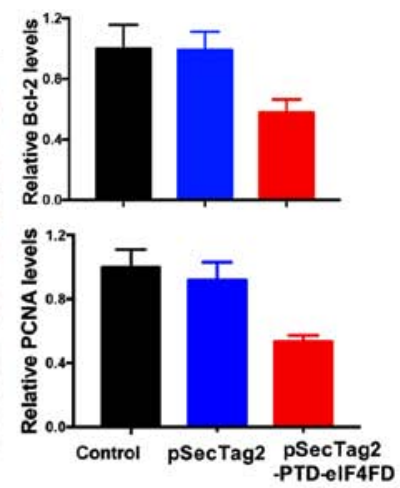

D

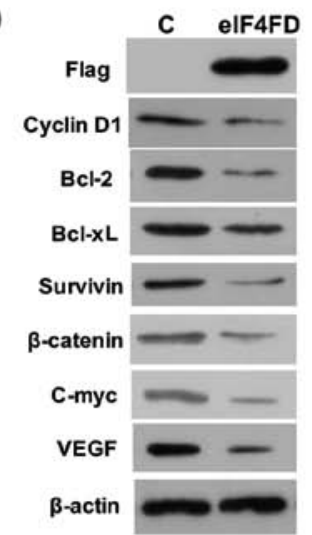

Figure 4. pSecTag2-PTD-eIF4FD injection via the tail vein represses glioma growth in vivo. pSecTag2-PTD-eIF4FD or pSecTag2 was injected via tail vein twice a week for 4 weeks. According to the above methods, (A) tumor growth was observed and measured, (B) expression levels of PCNA and Bcl-2 were detected using an immunohistochemical assay. (C) TUNEL staining. Experimental results were analyzed by image analysis and statistical analysis. Scale bar, $10 \mu \mathrm{m}$. (D) Indicated proteins in glioma graft tissues were determined by western blot analysis.

of the eIF4F complex can directly enhance the expression of Bcl-2, Bcl-xL, survivin and other anti-apoptotic proteins $(8,9)$. The knockdown or inhibition of survivin is beneficial in the treatment of glioblastoma (30). In the present study, TUNEL staining revealed that the number of positive U251 cells was significantly increased following pSecTag2-PTD-eIF4FD transfection. Accordingly, the protein levels of anti-apoptotic Bcl-2, Bcl-xL and survivin were all downregulated in the pSecTag2-PTD-eIF4FD-transfected cells. These results may explain the higher rates of apoptotic cell death, which was consistent with the previous studies. The results showed that the eIF4F complex-dependent initiation of translation is important in resistance to the apoptosis of cancer cells, suggesting that preventing eIF4F complex formation may promote apoptosis via the downregulation of $\mathrm{Bcl}-2, \mathrm{Bcl}-\mathrm{xL}$ and survivin proteins. Other studies have reported that eIF4F complex activation can directly promote the 5'-cap structure-dependent mRNA translation, including survivin and $\beta$-catenin (10), and the translation product $\beta$-catenin promotes the transcription of survivin (11). $\beta$-catenin can directly enhance the proliferation and survival of endothelial cells, but also promotes angiogenesis by inducing the expression of VEGF and promoting angiogenic progenitor cell mobilization (31). Western blot analysis showed that the expression levels of $\beta$-catenin and VEGF were decreased with the expression of eIF4FD. The results of the present study indicated that inhibiting eIF4F complex assembly also led to the inhibition of tumor angiogenesis by downregulating the expression of $\beta$-catenin and survivin.
To examine the application of the pSecTag2-PTD-eIF4FD vector as a tool for cancer therapy, the present study established an orthotopic xenograft model in nude mice. The data showed that the xenografts overexpressing eIF4FD showed a significant delay of growth rate and smaller tumor volumes, accompanied by a high rate of apoptosis. Furthermore, the injection of pSecTag2-PTD-eIF4FD via the tail vein into glioma xenograft mice also decreased cell growth rate and induced apoptosis, indicating a high-efficiency therapeutic potential on malignant gliomas in vivo. It has been shown that non-small cell lung cancer cells stably transfected with dominant active mutant 4E-BP1 $1^{\mathrm{A} 37 / \mathrm{A} 46}$ (HA-TTAA) suppressed growth and decreased tumorigenicity in xenograft models. Xenograft tumors expressing HA-TTAA were markedly smaller (32). Breast carcinoma xenografts in pSecX-t4EBP1 mice also exhibited significantly delayed growth and smaller tumor volume, with a higher tumor inhibition rate compared with the control and pSecX groups (33). The results of the present study were consistent with these findings.

In conclusion, the present study showed that the eukaryotic expression vector pSecTag2-PTD-eIF4FD, which contains the phosphorylation defective truncated 4E-BP2, effectively inhibited eIF4E and prevented eIF4F complex assembly by the expression of product PTD-eIF4FD. Inhibiting the eIF4F complex suppressed cell proliferation, induced apoptosis and inhibited glioma vascularization in vitro, and inhibited tumor growth and promoted tumor apoptosis in vivo. Therefore, it was concluded that gene therapy of malignant glioma through 
targeting the translation initiation complex eIF4F warrants further investigation.

\section{Acknowledgements}

Not applicable.

\section{Funding}

The present study was supported by a grant from the Natural Science Foundation of China (grand nos. 81172396, 2011 and 81472358, 2014).

\section{Availability of data and materials}

The datasets used and/or analyzed during the current study are available from the corresponding author on reasonable request.

\section{Authors' contributions}

QFD, ZFY and HNZ designed the research; QFD, ZFY, PQL, XY and JLH performed the research; JLH contributed the new reagents and analytic tools; QFD, ZFY and HNZ analyzed the data and wrote the manuscript. All authors read and approved the manuscript and agree to be accountable for all aspects of the research in ensuring that the accuracy or integrity of any part of the work are appropriately investigated and resolved.

\section{Ethics approval and consent to participate}

All procedures were in accordance with the NIH Guide and were approved by the Ethics Committee of the Fourth Military Medical University.

\section{Patient consent for publication}

Not applicable.

\section{Competing interests}

The authors declare that they have no competing interests.

\section{References}

1. Bastien JI, McNeill KA and Fine HA: Molecular characterizations of glioblastoma, targeted therapy, and clinical results to date. Cancer 121: 502-516, 2015.

2. Jane EP, Premkumar DR, Cavaleri JM, Sutera PA, Rajasekar T and Pollack IF: Dinaciclib, a Cyclin-Dependent Kinase inhibitor promotes proteasomal degradation of Mcl-1 and enhances ABT-737 mediated cell death in malignant human glioma cell lines. J Pharmacol Exp Ther 356: 354-365, 2016.

3. Rybalkina EY, Pavlova GV and Stavrovskaya AA: Recent news in the glioblastoma research. Biochem Moscow Suppl Ser A 9: $1-12,2015$.

4. Graff JR, Konicek BW, Carter JH and Marcusson EG: Targeting the eukaryotic translation initiation factor 4E for cancer therapy. Cancer Res 68: 631-634, 2008.

5. Hagner PR, Abraham S and Gartenhaus RB: Targeting the translational machinery as a novel treatment strategy for hematologic malignancies. Blood 115: 2127-2135, 2010.

6. Hsieh AC and Ruggero D: Targeting eukaryotic translation initiation factor 4E (eIF4E) in cancer. Clin Cancer Res 16: 4914-4920, 2010 .
7. Rhoads RE: eIF4E: New family members, new binding partners, new roles. J Biol Chem 284: 16711-16715, 2009.

8. Graff JR, Konicek BW, Vincent TM, Lynch RL, Monteith D, Weir SN, Schwier P, Capen A, Goode RL, Dowless MS, et al: Therapeutic suppression of translation initiation factor eIF4E expression reduces tumor growth without toxicity. J Clin Invest 117: 2638-2648, 2007.

9. Peponi E, Drakos E, Reyes G, Leventaki V, Rassidakis GZ and Medeiros LJ: Activation of mammalian target of rapamycin signaling promotes cell cycle progression and protects cells from apoptosis in mantle cell lymphoma. Am J Pathol 169: 2171-2180, 2006.

10. Karni R, Gus Y, Dor Y, Meyuhas O and Levitzki A: Active Src elevates the expression of beta-catenin by enhancement of cap-dependent translation. Mol Cell Biol 25: 5031-5039, 2005.

11. Torres VA, Tapia JC, Rodriguez DA, Lladser A, Arredondo C, Leyton L and Quest AF: E-cadherin is required for caveolin-1-mediated down-regulation of the inhibitor of apoptosis protein survivin via reduced beta-catenin-Tcf/Lef-dependent transcription. Mol Cell Biol 27: 7703-7717, 2007.

12. Lin CJ, Nasr Z, Premsrirut PK, Porco JA Jr, Hippo Y, Lowe SW and Pelletier J: Targeting synthetic lethal interactions between Myc and the eIF4F complex impedes tumorigenesis. Cell Rep 1: 325-333, 2012.

13. Boussemart L, Malka-Mahieu H, Girault I, Allard D, Hemmingsson $\mathrm{O}$, Tomasic $\mathrm{G}$, Thomas M, Basmadjian $\mathrm{C}$, Ribeiro N, Thuaud F, et al: eIF4F is a nexus of resistance to anti-BRAF and anti-MEK cancer therapies. Nature 513: 105-109, 2014.

14. Zhou $\mathrm{H}$ and Huang S: Role of mTOR signaling in tumor cell motility, invasion and metastasis. Curr Protein Pept Sci 12: 30-42, 2011.

15. Robichaud N and Sonenberg N: eIF4E and Its Binding Proteins In: Parsyan A (ed). Translat Regulat Cancer Biol Med. Springer, Dordrecht, pp73-113, 2014.

16. Satheesha S, Cookson VJ, Coleman LJ, Ingram N, Madhok B, Hanby AM, Suleman CA, Sabine VS, Macaskill EJ, Bartlett JM, et al: Response to mTOR inhibition: Activity of eIF4E predicts sensitivity in cell lines and acquired changes in eIF4E regulation in breast cancer. Mol Cancer 10: 19, 2011.

17. Ayuso MI, Martinez-Alonso E, Salvador N, Bonova P, Regidor I and Alcázar A: Dissociation of eIF4E-binding protein 2 (4E-BP2) from eIF4E independent of $\mathrm{Thr}^{37} / \mathrm{Thr}^{46}$ phosphorylation in the ischemic stress response. PLoS One 10: e0121958, 2015.

18. Lukhele S, Bah A, Lin H, Sonenberg N and Forman-Kay JD: Interaction of the eukaryotic initiation factor 4E with 4E-BP2 at a dynamic bipartite interface. Structure 21: 2186-2196, 2013.

19. Patel LN, Zaro JL and Shen WC: Cell penetrating peptides: Intracellular pathways and pharmaceutical perspectives. Pharm Res 24: 1977-1992, 2007.

20. Wagstaff KM and Jans DA: Protein transduction: Cell penetrating peptides and their therapeutic applications. Curr Med Chem 13: 1371-1387, 2006.

21. Ozawa T, Wang J, Hu LJ, Bollen AW, Lamborn KR and Deen DF: Growth of human glioblastomas as xenografts in the brains of athymic rats. In Vivo 16: 55-60, 2002.

22. Candolfi M, Curtin JF, Nichols WS, Muhammad AG, King GD, Pluhar GE, McNiel EA, Ohlfest JR, Freese AB, Moore PF, et al: Intracranial glioblastoma models in preclinical neuro-oncology: Neuropathological characterization and tumor progression. J Neurooncol 85: 133-148, 2007.

23. Doherty JE, Woodard LE, Bear AS, Foster AE and Wilson MH: An adaptable system for improving transposon-based gene expression in vivo via transient transgene repression. FASEB J 27: 3753-3762, 2013.

24. Wu M, Liu Y, Di X, Kang H, Zeng H, Zhao Y, Cai K, Pang T, Wang S, Yao Y and Hu X: EIF4E over-expresses and enhances cell proliferation and cell cycle progression in nasopharyngeal carcinoma. Med Oncol 30: 400, 2013.

25. Pettersson F, Del Rincon SV, Emond A, Huor B, Ngan E, Ng J, Dobocan MC, Siegel PM and Miller WH Jr: Genetic and pharmacologic inhibition of eIF4E reduces breast cancer cell migration, invasion, and metastasis. Cancer Res 75: 1102-1112, 2015.

26. Zhou FF, Yan M, Guo GF, Wang F, Qiu HJ, Zheng FM, Zhang Y, Liu Q, Zhu XF and Xia LP: Knockdown of eIF4E suppresses cell growth and migration, enhances chemosensitivity and correlates with increase in $\mathrm{Bax} / \mathrm{Bcl}-2$ ratio in triple-negative breast cancer cells. Med Oncol 28: 1302-1307, 2011. 
27. Campbell L, Jasani B, Griffiths DF and Gumbleton $M$ Phospho-4e-BP1 and eIF4E overexpression synergistically drives disease progression in clinically confined clear cell renal cell carcinoma. Am J Cancer Res 5: 2838-2848, 2015.

28. Yang X, Dong QF, Li LW, Huo JL, Li PQ, Fei Z and Zhen HN The cap-translation inhibitor 4EGI-1 induces mitochondrial dysfunction via regulation of mitochondrial dynamic proteins in human glioma U251 cells. Neurochem Int 90: 98-106, 2015.

29. Di Marino D, D'Annessa I, Tancredi H, Bagni C and Gallicchio E: A unique binding mode of the eukaryotic translation initiation factor 4E for guiding the design of novel peptide inhibitors. Protein Sci 24: 1370-1382, 2015.

30. Liao A, Shi R, Jiang Y, Tian S, Li P, Song F, Qu Y, Li J, Yun H and Yang X: SDF-1/CXCR4 axis regulates cell cycle progression and epithelial-mesenchymal transition via up-regulation of survivin in glioblastoma. Mol Neurobiol 53: 210-215, 2016.
31. Kim KI, Cho HJ, Hahn JY, Kim TY, Park KW, Koo BK, Shin CS $\mathrm{Kim} \mathrm{CH}$, Oh $\mathrm{BH}$, Lee MM, et al: Beta-catenin overexpression augments angiogenesis and skeletal muscle regeneration through dual mechanism of vascular endothelial growth factor-mediated endothelial cell proliferation and progenitor cell mobilization. Arterioscler Thromb Vasc Biol 26: 91-98, 2006.

32. Jacobson BA, Alter MD, Kratzke MG, Frizelle SP, Zhang Y, Peterson MS, Avdulov S, Mohorn RP, Whitson BA, Bitterman PB, et al: Repression of cap-dependent translation attenuates the transformed phenotype in non-small cell lung cancer both in vitro and in vivo. Cancer Res 66: 4256-4262, 2006.

33. Yang H, Li LW, Shi M, Wang JH, Xiao F, Zhou B, Diao LQ, Long XL, Liu XL and Xu L: In vivo study of breast carcinoma radiosensitization by targeting eIF4E. Biochem Biophys Res Commun 423: 878-883, 2012. 